\title{
Longitudinal Serology of SARS-CoV-2-Infected Individuals in India: A Prospective Cohort Study
}

Ramachandran Thiruvengadam, ${ }^{1}$ Souvick Chattopadhyay, ${ }^{1}$ Farha Mehdi, ${ }^{1}$ Bapu Koundinya Desiraju, ${ }^{1}$ Susmita Chaudhuri, ${ }^{1}$ Savita Singh, ${ }^{1}$ Vandita Bhartia, ${ }^{1}$ Pallavi Kshetrapal, ${ }^{1}$ Uma Chandra Mouli Natchu, ${ }^{2}$ Nitya Wadhwa, ${ }^{1}$ Shailaja Sopory, ${ }^{1}$ Mudita Wahi, ${ }^{1}$ Anil K. Pandey, ${ }^{3}$ Juhi Taneja, ${ }^{3}$ Nidhi Anand, ${ }^{3}$ Nandini Sharma, ${ }^{4}$ Pragya Sharma, ${ }^{4}$ Sonal Saxena, ${ }^{4}$ Deepa Sindhu, ${ }^{5}$ Brahmdeep Sindhu, ${ }^{6}$ Dharmendra Sharma, ${ }^{1}$ Tripti Shrivastava, ${ }^{1}$ Arjun Dang, ${ }^{7}$ Gaurav Batra, ${ }^{1}$ Gagandeep Kang, ${ }^{1,8 *} \dagger$ and Shinjini Bhatnagar ${ }^{1 *} \dagger$ for the DBT India Consortium for COVID 19 Research

${ }^{1}$ Translational Health Science and Technology Institute, Faridabad, Haryana, India; ${ }^{2}$ St. John's Medical College and St. John's Research Institute, Bengaluru, India; ${ }^{3}$ ESIC Medical College and Hospital, Faridabad, Haryana, India; ${ }^{4}$ Maulana Azad Medical College and Lok Nayak Hospital, New Delhi, India; ${ }^{5}$ Civil Hospital, Gurugram, India; ${ }^{6}$ Civil Hospital, Palwal, India; ${ }^{7}$ Dr. Dang's Laboratory, New Delhi, India; ${ }^{8}$ Christian Medical College, Vellore, India

\begin{abstract}
Clinical and epidemiological characteristics of severe acute respiratory syndrome coronavirus 2 (SARSCoV-2) are now widely available, but there are few data regarding longitudinal serology in large cohorts, particularly those from low-income and middle-income countries. We established an ongoing prospective cohort of 3,840 SARS-CoV-2positive individuals according to RT-PCR in the Delhi-National Capital Region of India to document clinical and immunological characteristics during illness and convalescence. The immunoglobulin $\mathrm{G}(\mathrm{IgG})$ responses to the receptor binding domain (RBD) and nucleocapsid were assessed at 0 to 7 days, 10 to 28 days, and 6 to 10 weeks after infection. The clinical predictors of seroconversion were identified by multivariable regression analysis. The seroconversion rates during the postinfection windows of 0 to 7 days, 10 to 28 days, and 6 to 10 weeks were $46 \%, 84.7 \%$, and $85.3 \%$, respectively ( $N=$ 743). The proportion with a serological response increased with the severity of coronavirus disease 2019 (COVID-19). All participants with severe disease, $89.6 \%$ with mild to moderate infection, and $77.3 \%$ of asymptomatic participants had lgG antibodies to the RBD antigen. The threshold values for the nasopharyngeal viral RNA RT-PCR of a subset of asymptomatic and symptomatic seroconverters were comparable $(P=0.48)$ to those of nonseroconverters $(P=0.16)(N=169)$. This is the first report of longitudinal humoral immune responses to SARS-CoV-2 over a period of 10 weeks in South Asia. The low seropositivity of asymptomatic participants and differences between assays highlight the importance of contextualizing the understanding of population serosurveys.
\end{abstract}

\section{INTRODUCTION}

Coronavirus disease 2019 (COVID-19), which is caused by severe acute respiratory syndrome coronavirus 2 (SARS-CoV$2)$, has emerged as one of the most significant global public health challenges of the 21 st century. The clinical phenotype of SARS-CoV-2 infection varies across a spectrum ranging from asymptomatic to mild (moderate) illness to severe COVID-19. The proportions of the specific phenotypes differ with geographical location. ${ }^{1}$ Prospectively followed cohorts of SARS-CoV-2-infected individuals have provided the opportunity to accurately describe the clinical characteristics of the disease in diverse geographical settings during different time periods of evolution of the pandemic. When combined with a systematic collection of biospecimens, such cohorts can serve as useful platforms to answer the emerging questions of importance to public health.

The characterization of immunological responses to SARSCoV-2 infection and its association with the clinical spectrum of disease is still evolving. Detection of anti-SARS-CoV-2 antibodies targeting nucleocapsid (NC) and spike protein, particularly the receptor binding domain (RBD) of the $S$ protein, is being used to evaluate serological humoral responses according to age and clinical phenotypes. Data regarding the persistence of antibodies against these proteins are

*Address correspondence to Shinjini Bhatnagar, Professor of Eminence and Head- Maternal and Child Health, Translational Health Science and Technology Institute, Faridabad, India, or Gagandeep Kang, Professor, The Wellcome Trust Research Laboratory, Division of Gastrointestinal Sciences, Christian Medical College, Vellore, TN 632004, India. E-mails: shinjini.bhatnagar@thsti.res.in or gkang@ cmcvellore.ac.in

†These authors contributed equally to this work. emerging, but few studies have been performed in lowincome and middle-income countries.

We measured immunoglobulin $\mathrm{G}$ ( $\mathrm{lgG}$ ) antibodies against SARS-CoV-2 RBD and NC protein in participants enrolled in a multihospital-based prospective cohort from northern India for up to at least 6 weeks postinfection and correlated the clinical and demographic differences between seroconverters and nonseroconverters.

\section{METHODS}

Study design and participants. This study was developed by interdisciplinary research institutes and hospitals in the National Capital Region of India. It was coordinated by the Translational Health Science and Technology Institute. The main clinical sites were ESIC Medical College Hospital, Faridabad, and Loknayak Hospital, New Delhi. The study protocol was approved by the Institute Ethics Committees of all participating institutions.

Cohort enrollment. During the ongoing cohort study, we enrolled COVID-19-positive patients across all ages tested at or admitted to designated COVID-19 testing centers or hospitals within 5 days of their positive RT-PCR test results. The eligibility criteria were positive RT-PCR results according to the National Testing Strategy of India and written informed consent. $^{2}$ The strategy for including participants from testing centers involved the enrollment of outpatients; however, inpatient recruitment was performed in hospital wards was to enrich the cohort with patients with COVID-19.

Follow-up. The follow-up visits were designed to capture the clinical outcomes of illness (10-28 days after being diagnosed with SARS-CoV-2) and the early (6-10 weeks) and late (6 and 12 months) convalescent periods (Supplemental 
TABLE 1

Demographic and clinical characteristics of participants

\begin{tabular}{|c|c|c|c|}
\hline \multirow[b]{2}{*}{ Baseline characteristics } & \multicolumn{2}{|r|}{ Full cohort $(N=2,504)$} & \multirow{2}{*}{$\begin{array}{l}\text { Participants included for serological evaluation } \\
(N=743)\end{array}$} \\
\hline & $\mathrm{N}$ & Median (IQR or IQR; $95 \% \mathrm{Cl}$ ) or $\mathrm{n}(\%$ or $\% ; 95 \% \mathrm{Cl})$ & \\
\hline Age, years & 2,452 & $42(29-55)$ & $40(29-53)$ \\
\hline Male & 2,504 & $1,703(68 \%)$ & $519(70 \%)$ \\
\hline Symptomatic & 2,504 & $2,078(83 \%)$ & 626 (84\%) \\
\hline Healthcare workers & 2,504 & 257 (10.3\%) & 57 (7.3\%) \\
\hline History of primary contact & 2,504 & & \\
\hline No & & $1,510(60 \%)$ & $441(59 \%)$ \\
\hline Not known & & 483 (19\%) & 139 (19\%) \\
\hline Yes & & $511(20 \%)$ & $163(22 \%)$ \\
\hline History of secondary contact & 2,504 & & \\
\hline No & & $1,551(62 \%)$ & $447(60 \%)$ \\
\hline Not known & & 889 (35\%) & 275 (37\%) \\
\hline Yes & & $64(2.6 \%)$ & $21(2.8 \%)$ \\
\hline Duration of contact, days & 386 & $4.0(2.0-7.8)$ & $4.0(2.0-8.0)$ \\
\hline Duration of contact, hours per day & 309 & $12(6-24)$ & $12(6-24)$ \\
\hline \multicolumn{4}{|l|}{ Outcome characteristics } \\
\hline Disease severity & 2,504 & & \\
\hline Asymptomatic & & $411(16 \% ; 14.98-17.92)$ & 199 (25.5\%; 22.42-28.65) \\
\hline Mild to moderate & & $1,638(65 \% ; 63.51-67.27)$ & $513(65.6 \% ; 62.15-68.93)$ \\
\hline Severe & & 455 (18\%; 16.67-19.73) & 70 (8.95\%; 7.0-11.17) \\
\hline Intensive care & 2,278 & $75(3.3 \% ; 2.59-4.10)$ & $24(3.1 \% ; 1.98-4.53)$ \\
\hline Oxygen therapy & 2,313 & 432 (19\%; 17.10-20.32) & $134(17 \% ; 14.55-19.96)$ \\
\hline Mechanical ventilation & 2,275 & $10(0.4 \% ; 0.21-0.80)$ & $2(0.3 \% ; 0.03-0.92)$ \\
\hline Death & 2,504 & $23(0.9 \% ; 0.58-1.37)$ & $0(0 \%)$ \\
\hline $\begin{array}{l}\text { Time to death from date of diagnosis, } \\
\text { days }\end{array}$ & 23 & $25(16-35)$ & NA \\
\hline $\begin{array}{l}\text { Time to death from date of onset of } \\
\text { symptoms, days }\end{array}$ & 20 & $34(20-43)$ & NA \\
\hline
\end{tabular}

Figure 1). The duration of illness was defined as the date of onset of symptoms for symptomatic participants; it was defined as the date of positive tests results indicating SARSCoV-2 infection among those who were asymptomatic. ${ }^{3}$ This report presents only data until early convalescence; later follow-up is still ongoing.

Clinical data and biospecimen collection. A trained research team collected clinical data and biospecimens at enrollment and follow-up. The demography and clinical characteristics focused on symptoms, comorbidities, drug history, and treatment details were collected by electronic data capture based on the standard operating protocol developed a priori. Venous blood samples were collected and transported according to biosafety protocols recommended by the Government of India and the World Health Organization. ${ }^{4}$ Serum was separated and stored in the biorepository at Translational Health Science and Technology Institute for subsequent analyses.

Serological assays. Anti-SARS-CoV-2 RBD lgG antibody was detected using an ELISA as described previously. ${ }^{5}$ Briefly, samples and controls were diluted to 1:50 in the sample diluent, and $100 \mu \mathrm{L}$ was added per well of the stabilized RBD-coated plate. After 30 minutes of incubation at room temperature $\left(23 \pm 2^{\circ} \mathrm{C}\right)$, the plates were washed six times with wash buffer. Then, $50 \mu \mathrm{L}$ of ready-to-use conjugate (horseradish peroxidase-labeled goat anti-human IgG Fcyspecific antibody) was added to each well and incubated for 30 minutes at room temperature, followed by six washes. Then, $100 \mu \mathrm{L}$ of substrate was added to each well and incubated at room temperature for 10 minutes; the reaction was stopped with $100 \mu \mathrm{L}$ of stop solution in each well. The absorbance was measured on a microplate reader at $450 \mathrm{~nm}$ with $650 \mathrm{~nm}$ as a reference wavelength. The pooled negative control and pooled positive control were performed on each plate. The cutoff for each plate was calculated by taking the average optical density of the triplicate of the negative control and adding 0.2 to this value. The signal-to-cutoff ratio (S/Co) was calculated as the ratio of the optical density value from the test sample to the cutoff value. An S/Co ratio $\geq 1$ was considered positive.

The anti-NC IgG chemiluminescence assay (SARS-CoV-2 IgG; Abbott Diagnostics Division, Sligo, Ireland) was performed according to the manufacturer's instructions; a calibrator was used and positive and negative controls were performed before each batch of antibody testing according to the manufacturer's protocol. The results were obtained by dividing the chemiluminescent signal from the sample by the mean of chemiluminescent signals from three calibrator replicates. The default result unit is index $(\mathrm{S} / \mathrm{Co})$; results higher than a cut-off of 1.4 were considered positive.

Statistical analysis. Seroconverters were defined as those whose test results were seropositive according to either assay at least once any time during the study period comprising 10 weeks of follow-up. The clinical phenotypes of COVID-19 were stratified as severe, mild to moderate, and asymptomatic based on their most severe symptoms and/or the need for treatment at enrollment or at any time during the follow-up period. Participants were considered to have severe COVID19 if they died or required oxygen supplementation or ventilatory support, or if they required treatment in the intensive care unit for cardiopulmonary or multiorgan dysfunction. Mild to moderate disease was defined by the presence of 
symptoms of COVID-19 that did not fulfill the criteria for severe disease. Those who did not report any symptoms at enrollment or throughout the follow-up period were classified as having asymptomatic COVID-19. The clinical and epidemiological characteristics of the participants are described as median and interquartile range (IQR) for continuous variables, and as percentages for categorical variables. The univariate analyses were performed using the $x^{2}$ test for comparison of proportions. The Mann Whitney $U$ test was performed to determine the differences in distributions between among clinical groups (seroconverters and nonseroconverters). A multivariable regression analysis was performed to identify independent predictors of seroconversion. We included all clinical variables collected during the study as independent variables in the multivariable regression analysis. The differences in cycle threshold values between asymptomatic and symptomatic individuals in both seroconversion and nonseroconversion categories were tested for significance using the Mann Whitney $U$ test. All statistical analyses were performed using $R$.

\section{RESULTS}

Baseline and clinical characteristics of the participants. The cohort had 3,790 COVID-19 patients on December 18, 2020; the majority of those enrolled were from Lok Nayak Hospital, New Delhi (45\%) and ESIC Medical College Hospital, Faridabad (44.6\%). We present the clinical results of the first 2,504 (2139 from the hospital and 365 from testing centers) participants enrolled between April 2020 and October 2020, that were obtained 6 to 10 weeks postdiagnosis. The median age was 44 years (IQR, 30-57), and more than two-thirds of the patients were male. Nearly two-thirds of the patients presented with symptoms; the most common symptoms were fever (61\%), cough (48\%), breathlessness $(35 \%)$, sore throat $(27 \%)$, and body ache $(22 \%)$. Only one out of five individuals had a history of primary contact defined as direct contact for 15 minutes without a mask with someone who had positive test results for COVID-19. A history of secondary contact, defined as direct contact for 15 minutes without a mask with someone who had a history of primary contact was noted for $2 \%$. Among those with a history of contact, the median duration since contact was 4 days (IQR, 2-7 days). The baseline characteristics of the participants who underwent serological analysis $(N=743)$ are provided in Table 1.

Serological characteristics. The longitudinal serological evaluation included participants who contributed all three samples $(N=743)$. The seroconversion rates during the windows of 0 to 7 days, 10 to 28 days, and 6 to 10 weeks were $46 \%, 84.7 \%$, and $85.3 \%$, respectively (Table 2) and Supplemental Table 1. The positive proportion increased with the severity of COVID-19; $100 \%$ with severe disease, $89.6 \%$ with mild to moderate disease, and $77.3 \%$ who were asymptomatic had IgG antibodies to the RBD antigen. As shown in Figure 1 and Table 3, the response to the NC antigen by the anti-NC chemiluminescence assay was lower (severe, 98.3\%; mild-moderate, 85.9\%; and asymptomatic, 69.9\%). Higher seroconversion with disease severity was consistent when we evaluated the S/Co ratio of the anti-RBD antibody and the anti-NC IgG antibody (Figure 2). Notably, those who were seropositive for anti-RBD lgG at enrollment had an almost $60 \%$ higher risk of severity (38/242 versus $29 / 294$; relative risk $[R R], 1.59$; 95\% confidence interval $[\mathrm{Cl}], 1.01-2.50)$ than 


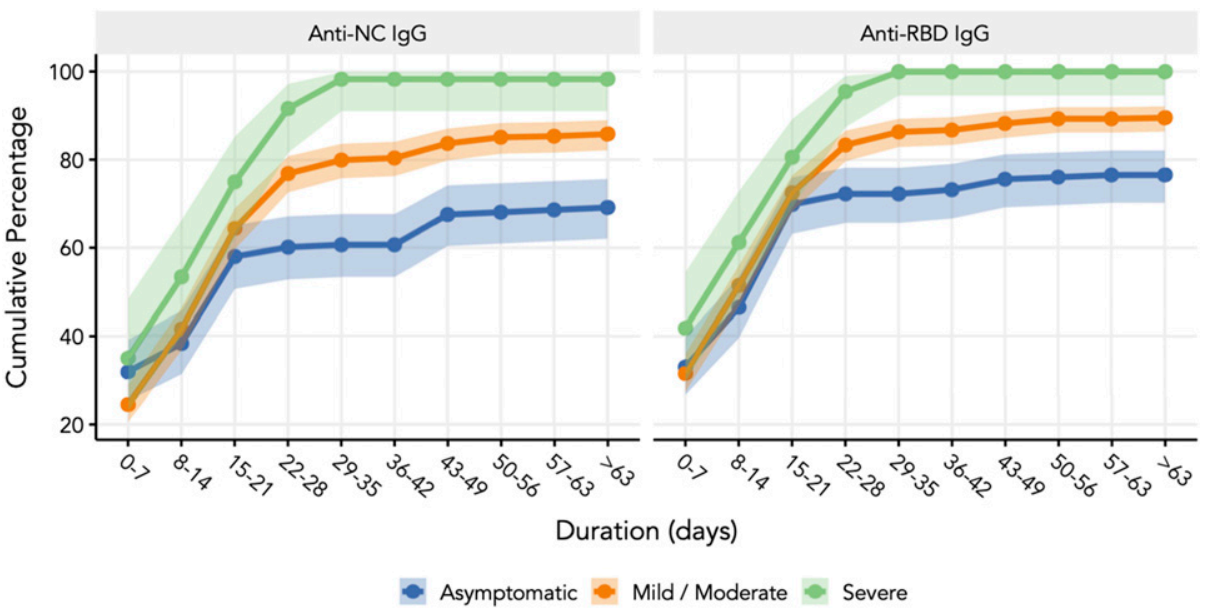

FIGURE 1. Cumulative seropositivity for anti-nucleocapsid (anti-NC) and anti-receptor binding domain (anti-RBD) immunoglobulin G (IgG) antibodies among different categories of disease severity. The error bars indicate the $95 \%$ confidence interval $(\mathrm{Cl})$ of the signal/cutoff ratio during that window of follow-up. This figure appears in color at www.ajtmh.org.

those who remained seronegative or became seropositive at a later time point. The threshold values of the nasopharyngeal viral RNA RT-PCR of asymptomatic and symptomatic seroconverters were comparable $(P=0.48)$, as were those of asymptomatic and symptomatic nonseroconverters $(P=0.16)$ $(N=169)$ (Supplemental Figure 2). For all those who had an IgG response, there was no demonstrable decline, as seen by the S/Co ratio during up to 10 weeks of follow-up.

Predictors of seroconversion. Demographic and clinical characteristics associated with seroconversion are described in Table 4. More than 95\% ( $N=105)$ of participants older than 60 years had positive IgG antibody results; this proportion decreased with younger age. Interestingly, participants who reported no primary contact or those who were uncertain about their contact history experienced seroconversion more often than those who had a history of exposure. Seroconverters reported a longer exposure duration per day than those who did not exhibit an IgG response (median exposure: 24 hours versus 8 hours) (Table 5). The presence of preexisting comorbidities such as chronic hypertension $(P<$ $0.001)$ and diabetes mellitus $(P<0.001)$ were associated with seroconversion (Table 4). The multivariable model after adjusting for confounders indicated that age (years) (adjusted odds ratio [aOR], $1.03 ; 95 \% \mathrm{Cl}, 1.02-1.05)$ and the presence of symptoms at presentation $(\mathrm{aOR}, 3.23 ; 95 \% \mathrm{Cl}, 2.01-5.17)$ were independent predictors of seroconversion.

\section{DISCUSSION}

Age and symptomatic status were independent positive predictors of seroconversion for our Indian cohort. The higher antibody response with severe COVID infection was expected. A severe infection indicated either a higher infectious dose or the spread of viruses beyond the respiratory tract. In both situations, the probability of the host immune response increases. ${ }^{6-9}$ Furthermore, longer exposure of the host immune system caused by prolonged severe COVID-19 infection may lead to the generation of polyfunctional T cells, which elicit a cytokine storm and an evident humoral response. ${ }^{10,11}$ Our findings differ from those of studies performed in western countries that showed much higher seroconversion rates among asymptomatic individuals. ${ }^{12-14}$ However, a recent study performed in Bangladesh reported lower seroconversion rates (45\%) among asymptomatic individuals. ${ }^{15}$ This raises the possibility that biological responses to SARS COV-2 may vary among different populations.

Age is a known strong predictor of severe COVID-19, and it is possible that systemic spread of infection, possibly because of impaired innate immune protection, occurs in elderly individuals. The presence of comorbidities, such as chronic hypertension and diabetes mellitus, was found to be associated with seroconversion by the unadjusted analysis; this relationship was probably confounded by the ages of the participants.

Nearly $15 \%$ of participants did not have IgG antibodies against both RBD and NC antigens. The trends of seropositivity were largely similar; anti-RBD IgG were detectable marginally earlier during the course of illness and in a larger proportion of individuals than anti-NC IgG. This was particularly noticeable among asymptomatic individuals. There could be many reasons for the poorer antibody response among asymptomatic individuals in our cohort. Unlike with severe infection, a possible lack of systemic spread of the virus in asymptomatic individuals

TABLE 3

Proportion of seroconverters among different categories of disease severity

\begin{tabular}{|c|c|c|c|c|}
\hline Type of assay & Asymptomatic, n (\%) & Mild to moderate, $\mathrm{n}(\%)$ & Severe, $n(\%)$ & $\begin{array}{c}\text { Proportion among all categories, } \mathrm{n} \\
(\%)\end{array}$ \\
\hline $\begin{array}{c}\text { Anti-RBD lgG } \\
(N=743)\end{array}$ & $\begin{array}{c}160 \text { (77.29\%) }(95 \% \mathrm{Cl}, \\
70.98-82.81)\end{array}$ & $\begin{array}{c}\text { 420; } 89.55 \%(95 \% \mathrm{Cl}: \\
86.42-92.17)\end{array}$ & $\begin{array}{l}67(100.00 \%)(95 \% \mathrm{Cl}, \\
94.64-100.00)\end{array}$ & $\begin{array}{c}647(87 \%)(95 \% \mathrm{Cl} \\
84.45-89.41)\end{array}$ \\
\hline $\begin{array}{l}\text { Anti-NC IgG } \\
\quad(N=673)\end{array}$ & $\begin{array}{c}130(69.89 \%)(95 \% \mathrm{Cl}, \\
62.75-76.39)\end{array}$ & $\begin{array}{c}364(85.85 \%)(95 \% \mathrm{Cl}, \\
82.16-89.02\end{array}$ & $\begin{array}{l}59(98.33 \%)(95 \% \mathrm{Cl} \\
91.06-99.96)\end{array}$ & $\begin{array}{l}553(82.16 \%)(95 \% \mathrm{Cl} \\
79.06-84.99)\end{array}$ \\
\hline
\end{tabular}

The last column indicates the overall seropositivity rates. Numbers in the parentheses denote the $95 \%$ confidence intervals of the percentage estimates of seroconverters. Participants had results for both anti-RBD and anti-NC assays across categories of disease. 


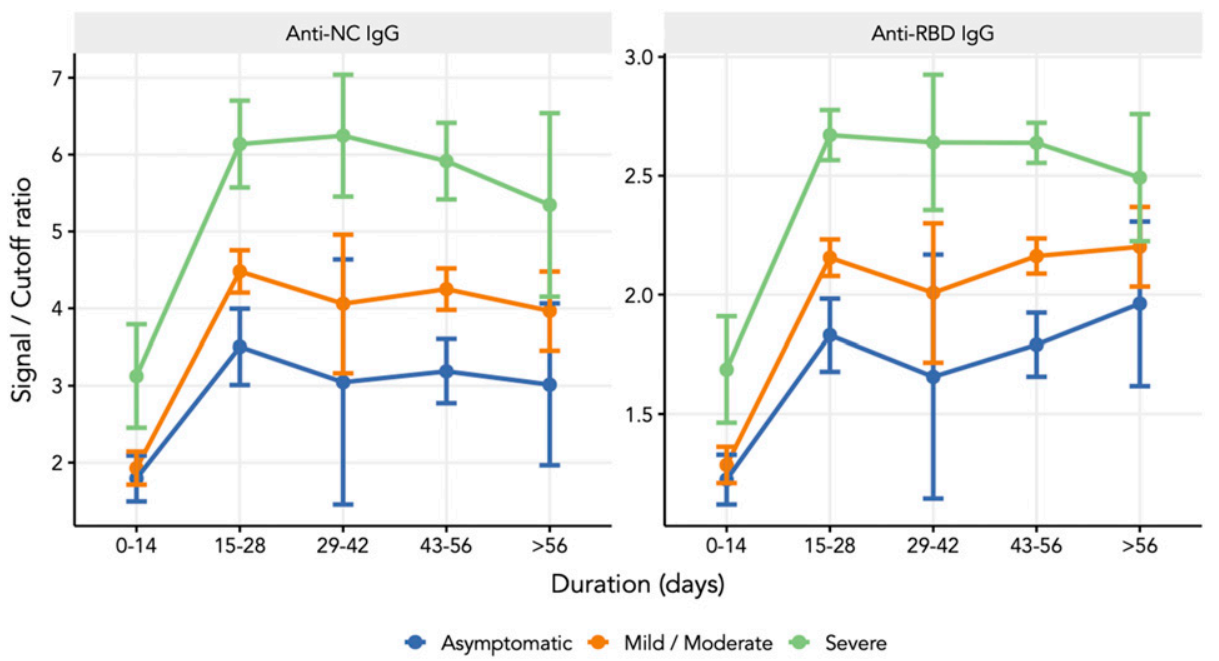

FIGURE 2. Longitudinal changes in the signal to cut-off ratios among different categories of disease severity. Anti-NC = anti-nucleocapsid; anti$\mathrm{RBD}=$ anti-receptor binding domain; IgG = immunoglobulin $\mathrm{G}$. This figure appears in color at www.ajtmh.org.

TABLE 4

Demographic and clinical characteristics associated with seroconversion $(N=743)$

\begin{tabular}{|c|c|c|c|c|}
\hline Characteristic & Total $\mathrm{N}$ of participants & Proportion of seroconverters, $\mathrm{n}(\%)$ & Unadjusted odds ratio $(95 \% \mathrm{Cl})$ & $P$ value \\
\hline \multicolumn{5}{|l|}{ Sex } \\
\hline Female & 212 & $176(83.02 \%)$ & \multirow[t]{2}{*}{$0.75(0.47-1.2)$} & \multirow[t]{2}{*}{0.24} \\
\hline Male & 513 & 445 (86.74\%) & & \\
\hline \multicolumn{5}{|c|}{ Symptom status at enrollment } \\
\hline Asymptomatic & 122 & $83(68.03 \%)$ & & \multirow[t]{2}{*}{$<0.001$} \\
\hline Symptomatic & 603 & $538(89.22 \%)$ & $3.88(2.38-6.29)$ & \\
\hline \multicolumn{5}{|l|}{ Age } \\
\hline 0-30 years & 190 & $143(75.26 \%)$ & & \multirow[t]{3}{*}{$<0.001$} \\
\hline $30-60$ years & 410 & $358(87.32 \%)$ & $2.26(1.42-3.59)$ & \\
\hline$>60$ years & 109 & 105 (96.33\%) & $8.58(3.00-33.81)$ & \\
\hline \multicolumn{5}{|l|}{ Comorbidities } \\
\hline \multicolumn{5}{|l|}{ Asthma } \\
\hline No & 708 & 609 (86.02\%) & & \multirow{2}{*}{0.15} \\
\hline Yes & 17 & 12 (70.59\%) & $0.39(0.12-1.45)$ & \\
\hline \multicolumn{5}{|l|}{ Autoimmune disorders } \\
\hline No & 721 & $618(85.71 \%)$ & & \multirow[t]{2}{*}{1.00} \\
\hline Yes & 4 & $3(75 \%)$ & $0.50(0.04-26.5)$ & \\
\hline \multicolumn{5}{|l|}{ Cancer } \\
\hline No & 715 & $612(85.59 \%)$ & \multirow{2}{*}{\multicolumn{2}{|c|}{$1.51(0.2167 .01)$}} \\
\hline Yes & 10 & $9(90 \%)$ & & \\
\hline \multicolumn{5}{|l|}{ Diabetes } \\
\hline No & 597 & $500(83.75 \%)$ & & \multirow[t]{2}{*}{$<0.001$} \\
\hline Yes & 128 & $121(94.53 \%)$ & $3.35(1.51-8.77)$ & \\
\hline \multicolumn{5}{|l|}{ Heart disease } \\
\hline No & 696 & 594 (85.34\%) & & \multirow[t]{2}{*}{0.37} \\
\hline Yes & 29 & $27(93.1 \%)$ & $2.32(0.57-20.4)$ & \\
\hline \multicolumn{5}{|l|}{ Hypertension } \\
\hline No & 605 & 505 (83.47\%) & & \multirow[t]{2}{*}{$<0.001$} \\
\hline Yes & 120 & $116(96.67 \%)$ & $5.73(2.10-21.89)$ & \\
\hline \multicolumn{5}{|l|}{ Kidney disease } \\
\hline No & 717 & $616(85.91 \%)$ & & \multirow[t]{2}{*}{0.17} \\
\hline Yes & 8 & 5 (62.5\%) & $0.27(0.05-1.79)$ & \\
\hline \multicolumn{5}{|l|}{ Liver disease } \\
\hline No & 717 & $615(85.77 \%)$ & & \multirow[t]{2}{*}{0.72} \\
\hline Yes & 8 & $6(75 \%)$ & $0.50(0.09-5.11)$ & \\
\hline More than one como & & & & \\
\hline No & 624 & 530 (84.94\%) & & 0.22 \\
\hline Yes & 101 & $91(90.1 \%)$ & $1.61(0.80-3.61)$ & \\
\hline Smoking & & & & \\
\hline No & 667 & 574 (86.06\%) & & 0.39 \\
\hline Yes & 58 & 47 (81.03\%) & $0.69(0.34-1.54)$ & \\
\hline Thyroid disorders & & & & \\
\hline No & 697 & 597 (85.65\%) & & 1.00 \\
\hline Yes & 28 & $24(85.71 \%)$ & $1.01(0.34-4.07)$ & \\
\hline
\end{tabular}


TABLE 5

Differences in the epidemiological characteristics of seroconverters and nonseroconverters

\begin{tabular}{|c|c|c|c|c|}
\hline Baseline characteristics & History of contact, $\mathrm{N}$ & Nonseroconverters, median (IQR) & Seroconverters, median (IQR) & $P$ value \\
\hline Duration of contact, days & 124 & $4.0(1.0-8.5)(N=31)$ & $4.0(2.0-7.0)(N=93)$ & 0.8 \\
\hline Duration of contact, hours per day & 99 & $8(2-24)(N=27)$ & $24(7-24)(N=72)$ & 0.01 \\
\hline $\begin{array}{l}\text { Duration between date of first contact and } \\
\text { date of diagnosis, days }\end{array}$ & 127 & $9(5-11)(N=33)$ & $6(3-10)(N=94)$ & 0.13 \\
\hline $\begin{array}{l}\text { Duration between date of last contact and } \\
\text { date of diagnosis, days }\end{array}$ & 122 & $1.0(-1.0$ to 6.0$)(N=31)$ & $1.0(-1.0$ to 4.0$)(N=91)$ & 0.6 \\
\hline
\end{tabular}

The history of contact was available for 185 of 743 participants. IQR = interquartile range.

would result in less exposure of the immune system. A large proportion of these individuals were identified by active contact tracing, and no or low seropositivity could reflect less exposure to a SARS-CoV-2-positive contact. ${ }^{16}$ It has been shown that the nasopharyngeal viral load is significantly higher among individuals with severe COVID-19 compared with that of individuals with mild illness. ${ }^{17}$ A small subset of individuals were seronegative until day 21 of their illness; however, they were seropositive at 6 to 10 weeks postinfection. This "negative-negative-positive" group of participants did not report any symptoms suggestive of SARS-CoV-2 infection; however, the possibility of having acquired a second asymptomatic infection during the follow-up period cannot be ruled out.

The lower seroconversion rates among asymptomatic RTPCR-positive participants are of critical significance to public health, particularly for the interpretation of community seroprevalence in highly affected geographical areas. Results of seroprevalence studies performed in India and other South Asian countries may need to be adjusted after considering our findings. ${ }^{18,19}$

The S/Co ratio for both assays reached the maxima between 15 and 28 days and plateaued, suggesting a stable lgG response during up to 10 weeks of illness. The longevity of the IgG response has been reported to vary between 5 weeks to 4 months. ${ }^{13,20-24}$ As shown by another global study, asymptomatic participants in our study did not show any decline in the $\mathrm{lgG}$ response to either antigen during a period of 10 weeks. ${ }^{25}$ This is in contrast to the initial evidence that showed early decline in the IgG response in asymptomatic infected individuals. ${ }^{3,13}$ We will follow-up the participants in this cohort for at least 1 year to evaluate the longevity of the lgG response.

The major strengths of the study are the prospective data collection, high follow-up rates, and the use of two validated antibody assays. However, there are some limitations. Because of the lack of robust IgM and lgA assays, we were unable to evaluate the immune response to these isotypes. Furthermore, because of the heterogeneity of the RT-PCR assays used by the participating hospitals for molecular diagnosis, we do not have comparable viral load data for all participants in our study. However, an analysis of a subset of the individuals who had similar RT-PCR assay results showed no differences in nasopharyngeal viral loads between seroconverters and nonseroconverters. The $95 \% \mathrm{Cl}$ for the association between the presence of anti-RBD IgG antibodies at enrollment and the severity of COVID-19 is wide, with the lower bound of the interval close to 1, suggesting the need for a larger sample size to confirm the association.

We systematically reported clinical and epidemiological characteristics of and longitudinal humoral immune responses to SARS-CoV-2 infection by a large Indian cohort. We will continue to study the kinetics of the immune response in a unique platform to evaluate the more complex and emerging questions regarding cellular immunity, reinfections, long COVID syndrome, and population-level postimmunization surveillance when the cohort participants are immunized.

Received February 10, 2021. Accepted for publication April 19, 2021. Published online May 18, 2021.

Note: Supplemental tables and figure appear at www.ajtmh.org.

Members of DBT India Consortium for COVID-19 Research:

Coordinating Institute: Translational Health Science and Technology Institute (THSTI)

Coordinating Principal Investigator: Dr. Shinjini Bhatnagar

Co-Principal Investigator: Dr. Gagandeep Kang

Co-Investigators (Clinical): Drs. Nitya Wadhwa, Uma Chandra Mouli Natchu, Ramachandran Thiruvengadam, Shailaja Sopory, Pallavi Kshetrapal, Bapu Koundinya Desiraju, Vandita Bhartia, Mudita Gosain, Monika Bahl

Co-Investigators (Laboratory): Drs. Gaurav Batra, Guruprasad Medigeshi, Susmita Chaudhuri, Niraj Kumar, Tarun Sharma, Chandresh Sharma, Shailendra Mani, Tripti Shrivastava

International Centre for Genetic Engineering and Biotechnology (ICGEB): Dr. Anmol Chandele

National Institute of Immunology (NII): Drs. Amulya Panda, Nimesh Gupta

Maulana Azad Medical College and Lok Nayak Hospital, New Delhi: Drs. Nandini Sharma, Pragya Sharma, Sonal Saxena, J. C. Passey, Suresh Kumar

ESIC Medical College and Hospital, Faridabad, Haryana: Dr. Anil K Pandey, Asim Das, Nikhil Verma, Nidhi Anand, Sujata Roy Choudhary, Juhi Taneja

Civil Hospital Gurugram (GCH), Haryana: Drs. Deepa Sindhu, Jai Singh Malik

Civil Hospital Palwal (PCH), Haryana: Dr. Brahmdeep Sindhu

Al-Falah School of Medical Science \& Research Centre and Hospital, Dhouj, Haryana: Drs. Bhupinder Kaur Anand, Shubham Girdhar

Medanta Hospital, Gurugram, Haryana: Drs. Sushila Kataria, Pooja Sharma

Shaheed Hasan Khan Mewati Government Medical College, Nalhar, Nuh, Haryana: Dr. Yamini

Lady Hardinge Medical College, New Delhi: Drs. Harish K. Pemde, Tanmaya Talukdar

SGT Medical college, Gurugram, Haryana: Drs. Pankaj Abrol, Mukesh Sharma

Dr. Dang's Lab, New Delhi: Drs. Navin Dang, Manavi Dang, Arjun Dang, Leena Chatterjee, Devjani De

Acknowledgments: We thank the Department of Biotechnology, Government of India, for supporting the consortium. We are grateful to the leadership and administration of all partner institutions in the consortium for their help and support. We thank all the clinical, 
laboratory, and data management staff for their contributions to this work and the consortium at large. The American Society of Tropical Medicine and Hygiene has waived the Open Access fee for this article due to the ongoing COVID-19 pandemic.

Financial support: This work was supported by Department of Biotechnology, Government of India (BT/PR40401/BIOBANK/03/2020), and by the Ind-CEPI Coalition for Epidemic Preparedness Innovations (102/IFD/SAN/5477/2018-2019).

Authors' addresses: Ramachandran Thiruvengadam, Souvick Chattopadhyay, Farha Mehdi, Bapu Koundinya Desiraju, Susmita Chaudhuri, Savita Singh, Vandita Bhartia, Pallavi Kshetrapal, Nitya Wadhwa, Dharmendra Sharma, Gaurav Batra, Shailaja Sopory, Mudita Gosain, and Shinjini Bhatnagar, Translational Health Science and Technology Institute, Faridabad, Delhi NCR, India, E-mails: ramachandran@thsti. res.in, ouvick@thsti.res.in, farha.mehdi@thsti.res.in, bapukoundinyadesiraju@ thsti.res.in, susmita@thsti.res.in, savitasingh@thsti.res.in, vanditabhartia@ gmail.com, pallavi.kshetrapal@thsti.res.in, nitya.wadhwa@thsti.res.in dksharma@thsti.res.in, gaurav.batra@thsti.res.in, mouli.natchu@sji.res.in, ssopory@thsti.res.in, mgosain@thsti.res.in, and shinjini.bhatnagar@ thsti.res.in. Anil K. Pandey, Juhi Taneja, and Nidhi Anand, ESIC Medical College and Hospital, Faridabad, Delhi NCR, India, E-mails: registraracademicfbd@gmail.com, drjuhitaneja@gmail.com, and nidhianand28@gmail.com. Nandini Sharma, Pragya Sharma, and Sonal Saxena, Maulana Azad Medical College and Lok Nayak Hospital, New Delhi, India, E-mails: drnandini1@gmail.com, drpragyas@ gmail.com, and sonalsaxena3@gmail.com. Deepa Sindhu and Brahmdeep Sindhu, Civil Hospital, Palwal and Gurugram, India, E-mails: deepajakhar9@gmail.com and bdsindhu@gmail.com. Navin Dang, Dr. Dang's Laboratory, New Delhi, India, E-mail: drnavindang@gmail.com. Gagandeep Kang, Christian Medical College, Vellore, India, E-mail: gkang@cmcvellore.ac.in.

This is an open-access article distributed under the terms of the Creative Commons Attribution (CC-BY) License, which permits unrestricted use, distribution, and reproduction in any medium, provided the original author and source are credited.

\section{REFERENCES}

1. Docherty $A B$ et al., 2020. Features of 20133 UK patients in hospital with COVID-19 using the ISARIC WHO Clinical Characterisation Protocol: prospective observational cohort study. BMJ 369: m1985.

2. Indian Council of Medical Research, 2020. Testing Strategy. Available at: https://www.icmr.gov.in/cteststrat.html.

3. Röltgen $\mathrm{K}$ et al., 2020. Defining the features and duration of antibody responses to SARS-CoV-2 infection associated with disease severity and outcome. Sci Immunol 5: eabe0240.

4. Ministry of Health and Family Welfare, Government of India, 2019. Sample collection packaging. Available at: https://www. mohfw.gov.in/pdf/5Sample\%20collection_packaging\%20\% 202019-nCoV.pdf.

5. Mehdi $F$ et al., 2021. Development of a fast SARS-CoV-2 IgG ELISA, based on receptor-binding domain, and its comparative evaluation using temporally segregated samples from RT-PCR positive individuals. Front Microbiol 11: 618097.

6. Liu Y, Yan LM, Wan L, Xiang TX, Le A, Liu JM, Peiris M, Poon LLM, Zhang W, 2020. Viral dynamics in mild and severe cases of COVID-19. Lancet Infect Dis 20: 656-657 (Epub 2020 Mar 19).

7. Chen $X$ et al., 2020. Detectable serum severe acute respiratory syndrome coronavirus 2 viral load (RNAemia) is closely correlated with drastically elevated interleukin 6 level in critically ill patients with coronavirus disease 2019. Clin Infect Dis 71: 1937-1942.

8. Eberhardt KA et al., 2020. RNAemia corresponds to disease severity and antibody response in hospitalized COVID-19 patients. Viruses 12: 1045.

9. Hogan CA et al., 2020. High frequency of SARS-CoV-2 RNAemia and association with severe disease. Clin Infect Dis ciaa1054 (Online ahead of print, doi: 10.1093/cid/ciaa1054).

10. Mazzoni A et al., 2020. Cell-mediated and humoral adaptive immune responses to SARS-CoV-2 are lower in asymptomatic than symptomatic COVID-19 patients. Eur J Immunol 50: 2013-2024 (Epub 2020 Nov 9).

11. Liu Y, Yan LM, Wan L, Xiang TX, Le A, Liu JM, Peiris M, Poon LLM, Zhang W, 2020. Viral dynamics in mild and severe cases of COVID-19. Lancet Infect Dis 20: 656-657 (Epub 2020 Mar 19).

12. Choe PG et al., 2020. Antibody responses to SARS-CoV-2 at 8 weeks postinfection in asymptomatic patients. Emerg Infect Dis 26: 2484-2487 (Epub 2020 Jun 24).

13. Long QX et al., 2020. Clinical and immunological assessment of asymptomatic SARS-CoV-2 infections. Nat Med 26: 1200-1204.

14. Chen $X$ et al., 2020. Disease severity dictates SARS-CoV-2specific neutralizing antibody responses in COVID-19. Signal Transduct Target Ther 5: 180.

15. Shirin T et al., 2020. Antibody responses after COVID-19 infection in patients who are mildly symptomatic or asymptomatic in Bangladesh. Int J Infect Dis 101: 220-225 (Epub 2020 Oct 5).

16. Wilder-Smith $A$, Teleman MD, Heng BH, Earnest A, Ling AE, Leo YS, 2005. Asymptomatic SARS coronavirus infection among healthcare workers, Singapore. Emerg Infect Dis 11: 1142-1145.

17. Liu Y, Yan LM, Wan L, Xiang TX, Le A, Liu JM, Peiris M, Poon LLM, Zhang W, 2020. Viral dynamics in mild and severe cases of COVID-19. Lancet Infect Dis 20: 656-657.

18. Murhekar MV et al., 2020. Prevalence of SARS-CoV-2 infection in India: findings from the national serosurvey, May-June 2020. Indian J Med Res 152 (1 \& 2): 48-60.

19. Uyoga $S$ et al., 2021. Seroprevalence of anti-SARS-CoV-2 IgG antibodies in Kenyan blood donors. Science 371: 79-82.

20. Younas A, Waheed S, Khawaja S, Imam M, Borhany M, Shamsi T, 2020. Seroprevalence of SARS-CoV-2 antibodies among healthy blood donors in Karachi, Pakistan. Transfus Apheresis Sci 59: 102923.

21. Long QX et al., 2020. Antibody responses to SARS-CoV-2 in patients with COVID-19. Nat Med 26: 845-848 (Epub 2020 Apr 29).

22. Wang $Y$ et al., 2020. Kinetics of viral load and antibody response in relation to COVID-19 severity. J Clin Invest 130: 5235-5244.

23. Kutsuna S, Asai Y, Matsunaga A, 2020. Loss of anti-SARS-CoV-2 antibodies in mild COVID-19. N Engl J Med 383: 1695-1696 (Epub 2020 Sep 23).

24. Ibarrondo FJ, Fulcher JA, Goodman-Meza D, Elliott J, Hofmann C, Hausner MA, Ferbas KG, Tobin NH, Aldrovandi GM, Yang OO, 2020. Rapid decay of anti-SARS-CoV-2 antibodies in persons with mild COVID-19. N Engl J Med 383: 1085-1087.

25. Gudbjartsson DF et al., 2020. Humoral immune response to SARS-CoV-2 in Iceland. N Engl J Med 383: 1724-1734. 